\title{
Molecular and Biochemical Examination of Spraing Disease in Potato Tuber in Response to Tobacco rattle virus Infection
}

\author{
Ghulam Sahi, ${ }^{1}$ Pete E. Hedley, ${ }^{1}$ Jenny Morris, ${ }^{1}$ Gary J. Loake, ${ }^{2}$ and Stuart A. MacFarlane ${ }^{1}$ \\ ${ }^{1}$ The James Hutton Institute, Invergowrie, Dundee DD2 5DA, U.K.; and ${ }^{2}$ Institute of Molecular Plant Sciences, School of \\ Biological Sciences, Kings Buildings, Mayfield Road, Edinburgh EH9 3JH, U.K.
}

Accepted 15 August 2016.

\begin{abstract}
Field-grown tubers of potato were examined for infection by Tobacco rattle virus (TRV) and consequent production of corky ringspot or spraing symptoms. A microarray study identified genes that are differentially expressed in tuber tissue in response to TRV infection and to spraing production, suggesting that hypersensitive response (HR) pathways are activated in spraing-symptomatic tubers. This was confirmed by quantitative reverse transcription-polymerase chain reaction (qRT-PCR) of a selected group of HR-related genes and by histochemical staining of excised tuber tissue with spraing symptoms. qRTPCR of TRV in different regions of the same tuber slice showed that nonsymptomatic areas contained higher levels of virus relative to spraing-symptomatic areas. This suggests that spraing formation is associated with an active plant defense that reduces the level of virus in the infected tuber. Expression of two of the same plant defense genes was similarly upregulated in tubers that were infected with Potato mop-top virus, a virus that also induces spraing formation.
\end{abstract}

Potato (Solanum tuberosum) is one of the world's most important food crops and is grown in diverse habitats across the globe. The potato tuber develops from the stolon, an outgrowth from the stem, which extends downward into the soil and expands as it accumulates starch and other nutrients. At a later time, sprouts can emerge from the tuber, which grow upwards to form the aerial parts of a new plant. Cultivated potatoes are grown clonally, from tuber to tuber, and not by collection and planting of true seed. However, the tubers that are selected for replanting to establish a new crop are referred to as seed potatoes.

One of the most important viral pathogens of potato is Tobacco rattle virus (TRV). This virus has a world-wide occurrence and an extremely wide host range (Harrison and Robinson, 1978; MacFarlane 1999). TRV is spread between plants primarily by soil-inhabiting nematodes of the genera Trichodorus and Paratrichodorus (collectively referred to as trichodorids). The

Data from these experiments has been deposited in the ArrayExpress databaseunder accession number E-MTAB-4670.

Corresponding author: S. A. MacFarlane; E-mail: s.macfarlane@hutton.ac.uk; Telephone: +44 (0)844 9285428; Fax: +44 (0)844 9285429

*The $\boldsymbol{e}$-Xtra logo stands for "electronic extra" and indicates that two supplementary figures, one supplementary table, and one supplementary data file are published online.

(C) 2016 The American Phytopathological Society nematodes acquire TRV while feeding on roots of infected plants and the virus particles adhere to particular locations of the nematode esophagus. Subsequent feeding events can release the virus particles into new plants to spread the infection. In addition, for potato, planting TRV-infected tubers can lead to infection in the aerial parts of the newly sprouted plants and also into the daughter tubers produced by these plants. For this reason, a key strategy to reduce TRV infection in potato crops is to ensure that the seed tubers are themselves free from TRV infection.

Infection of some potato cultivars by TRV leads to the production of arcs and flecks of discoloration in the tubers. These symptoms are referred to as corky ringspot (CRS) in the United States and spraing in the United Kingdom and mainland Europe (Harrison and Robinson 1978; Mojtahedi et al. 2001). In severe infections, the tuber symptoms can be accompanied by necrotic ring formation in the outer skin of the tuber. Both types of symptom can make the tuber unmarketable.

Despite spraing being an economically important disease of potato, there are very few research studies that have used molecular methods to investigate the infection of this crop plant by TRV (Crosslin et al. 1999; Ghazala and Varrelmann 2007; Harrison and Robinson 1982). One reason for this is the practical difficulty of producing a TRV infection in potato, for experimentation. Mechanical inoculation of TRV to the leaves of commercial varieties of tetraploid potato plants leads, usually, to a localized, low-level infection often accompanied by necrosis of the inoculated leaf and petiole (Ghazala and Varrelmann 2007; S. A. MacFarlane and G. Sahi, unpublished). Systemic spreading of TRV occurs infrequently and with such low levels of virus that this method of infection cannot be used to study tuber disease. Similarly, there are no reports in the literature of potato plants being successfully infected by manual inoculation of TRV to underground tissues (roots, stolons, or tubers).

Infection can be achieved by growing susceptible tubers in field soil that contains trichodorid nematodes carrying TRV. A major complication is that different nematode species transmit different TRV isolates (Ploeg et al. 1992a and b; Vassilakos et al. 2001), and for many TRV isolates, the nematode vector species is not known. TRV has two genome components, RNA1 and RNA2, with RNA1 encoding proteins involved in plant infection and RNA2 encoding the virus coat protein and nematode transmission-associated proteins. RNA1 can infect plants in the absence of RNA2, producing a so-called NM infection that has been found in stems and leaves of potato plants emerging from infected tubers. RNA1 is conserved in sequence between different isolates so that a generic TRV RNA1-specific reverse transcription-polymerase chain reaction (RT-PCR) detection test 
is available. In contrast, there is a huge variation in the sequences and genome organization of RNA2 from different TRV isolates. The complete sequences of 42 TRV RNA2 molecules have been deposited in GenBank and, apart from the 5' and 3' terminal regions, many isolates do not share any conserved sequences. This has meant that a generic RT-PCR detection test for TRV RNA2 has not been developed. This extreme variation also means that the coat proteins of the different TRV isolates are also very variable and no universal antibody is available for detection of all TRV isolates.

Our work has been done using potato tubers harvested from commercial fields in which TRV has previously been detected. This provided us with sufficient samples of both spraing-affected and spraing-free tubers but meant that we had no control over the growing conditions of each tuber or of the particular isolate of TRV that each tuber was infected by.

With this material, we have used microarray analysis to reveal the pattern of potato gene expression that occurs in spraing tissue from virus-infected tubers. We have also examined how expression levels of some spraing-associated potato genes correlates with TRV infection and investigated the distribution and relative levels of TRV in different areas of a spraing-affected potato tuber.

\section{RESULTS}

\section{Microarray and quantitative (q)RT-PCR studies.}

A microarray analysis of gene expression in mature tubers of potato cv. Pentland Dell was done. Four tubers from different plants showing spraing symptoms and containing TRV (samples S1 to S4), four tubers without visible spraing but containing TRV (SF1 to SF4) and four tubers without spraing or TRV (H1 to H4) were analyzed separately. As these tubers were harvested from the field rather than from a controlled-environment chamber, there were significant differences in the absolute gene expression measurements taken from each tuber. Nevertheless, the relative patterns of gene expression were consistent within each tuber type (symptomatic vs. spraing free vs. healthy), as was demonstrated by condition cluster analysis, whereby the majority of the biological replicates for each condition grouped together (Supplementary Fig. S1).

Across the experiment, reliable gene expression was detected from 27,895 probes on the array. Pairwise comparisons were performed to ascertain the relative differential gene expression between the sets of samples; in one analysis, spraing versus nospraing (spraing-free) samples and, in a second analysis, healthy versus TRV-infected spraing-free samples were compared. The comparisons, performed at a high stringency with a Student's $t$ test $P$ value restriction of 0.01 , identified 844 differentially expressed (greater than twofold difference) probes when comparing spraing versus spraing-free samples (both containing TRV) and 1,024 probes when comparing healthy versus TRVinfected but spraing-free samples. Lastly, comparing healthy versus spraing symptomatic samples, 2,827 differentially expressed probes were identified. The raw data from these experiments has been deposited in the ArrayExpress database (accession number E-MTAB-4670) and differentially expressed gene lists are shown in the Supplementary Data File.

When we examined the differentially expressed genes and compared the lists for all three sample-type comparisons, 630 probes (17.8\% of total number) were associated with spraing formation (Fig. 1). Likewise, 439 probes (12.4\% of total number) were associated with TRV infection, regardless of whether there was or was not spraing symptom production in the tuber.

The potato genes identified as being differentially expressed during these experiments were allocated to three overarching functional domains, according to Gene Ontology (GO) annotation. This analysis creates subsets of genes that are related by their expected GO terms, with the highest level domains being Molecular Function (GO: 3674), Biological Process (GO: 8150), and Cellular Component (GO: 5575). Molecular Function relates to genes involved in catalytic or enzymatic activities. Cellular Component relates to the subcellular location where the gene is active. Biological Process refers to a broad chain of events, comprised of single or multiple gene pathways, such as signal transduction or responses to biotic or abiotic stresses, and includes many lower-level GO term categories relevant to programmed cell death (PCD) and associated hypersensitive response (HR) reactions.

For the 844 probes differentially expressed in the spraing versus spraing-free samples, only 512 have a predicted function and only 495 have an ascribed GO term annotation. For the 1,024 probes differentially expressed in the healthy versus TRVinfected but spraing-free samples, only 585 have a predicted function and only 422 have an ascribed GO term annotation.

The virus-infected, spraing versus spraing-free expression analysis highlighted genes within several HR-related GO groups (GO: 6952 [defense response], GO: 6979 [response to oxidative stress], GO: 6800 [oxygen and reactive oxygen species metabolism], GO: 302 [response to reactive oxygen species], and GO: 42542 [response to hydrogen peroxide]. Also, several HR-related GO groups were highlighted from the comparison of healthy versus TRV-infected spraing-free samples (GO: 45454 [cell redox homeostasis], GO: 6800 [oxygen and reactive oxygen species metabolism], and GO: 9615 [response to virus]. This analysis revealed that genes within these ontologies were overrepresented in the complete list of differentially expressed genes from both the spraing versus spraing-free comparison and the healthy versus spraing-free comparison (Supplementary Table S1).

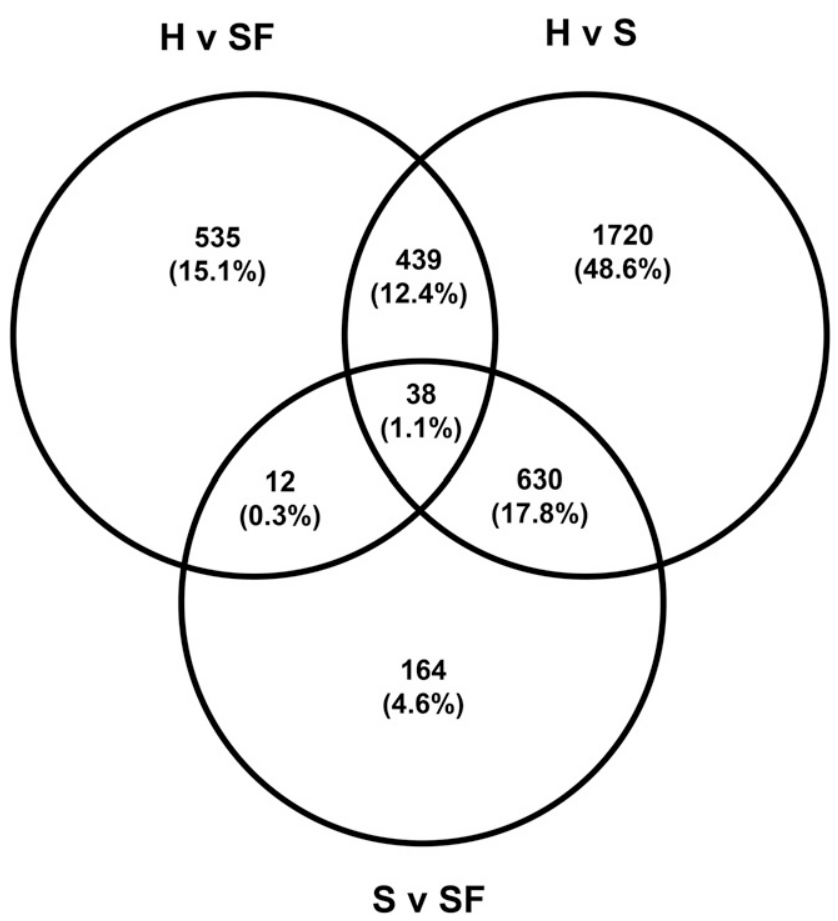

Fig. 1. Numbers and percentages of differentially (more than twofold) expressed genes in datasets. Comparisons are healthy $(\mathrm{H})$ versus virusinfected, spraing free (SF) samples; virus-infected, spraing symptomatic (S) versus SF; H versus $\mathrm{S}$. Regions of overlap denote differentially expressed genes shared between dataset comparisons. 
To validate the differences in gene expression identified during the microarray study, we carried out quantitative RTPCR (qRT-PCR) analysis of a subset of five genes selected from the HR-related ontologies described above and predicted from the microarray to have altered expression within the samples.

Initially, all four TRV-infected spraing-free (SF1 to SF4), all four TRV-infected, spraing symptomatic (S1 to S4), and all four healthy tuber (H1 to H4) RNA samples from the microarray experiments were examined by qRT-PCR to determine the relative amounts of TRV present in each sample. From this, the tuber samples with the highest levels of TRV infection (S1, S4, SF3, and SF4) and two of the healthy (TRV-free) tuber samples $(\mathrm{H} 1$ and $\mathrm{H} 2)$ were selected for qRT-PCR of HR-related potato tuber genes. We did initial experiments using both EF1 $\alpha$ and cyclophilin as endogenous comparators for normalization of the qRT-PCR data (data not shown). The relative levels of expression of the induced, HR-related genes was similar regardless of whether EF1 $\alpha$ or cyclophilin was used as the comparator; however, the cyclophilin gene was shown to be a more sensitive comparator and was used for most of the qRT-PCR experimentation in this study. The results of these analyses are shown in Figure 2A to E. The qRT-PCR experiments confirmed an upregulation of expression of each of these genes (encoding peroxidase, photoassimilate response 1-c [PAR1c], suberization-associated anionic peroxidase 2 [SP2], glutathione- $S$-transferase, respiratory burst oxidase homolog protein $\mathrm{B}$ ), thereby validating the overexpression data produced from the microarray experiments. This is the first experimental demonstration at the transcriptional level of the involvement of HR-related responses in the reaction of potato tubers to TRV infection and in the production of spraing symptoms.

\section{Tissue staining for $\mathrm{HR}$ reactions in tubers.}

No previous published work has examined spraing disease at the histological level; therefore, we applied tissue-staining techniques to excised sections of 'Pentland Dell' potato tubers exhibiting spraing symptoms. Three separate stains were applied: i) 3,3'-diaminobenzidine (DAB), which is used for the visualization of hydrogen peroxide $\left(\mathrm{H}_{2} \mathrm{O}_{2}\right)$ production and accumulation by the action of peroxidases to produce an insoluble
A

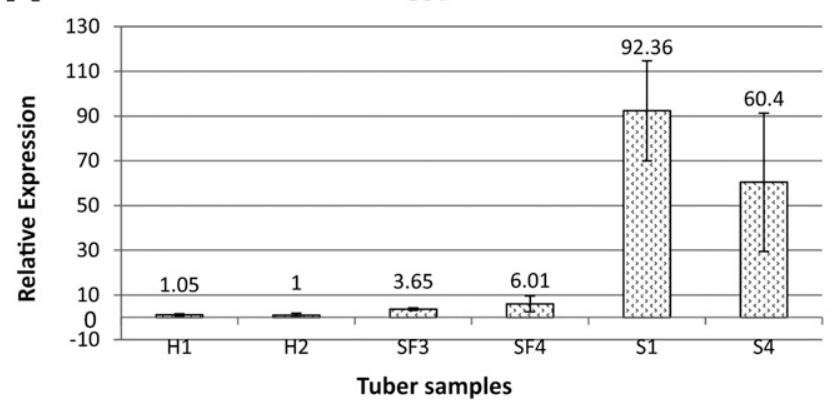

C SP

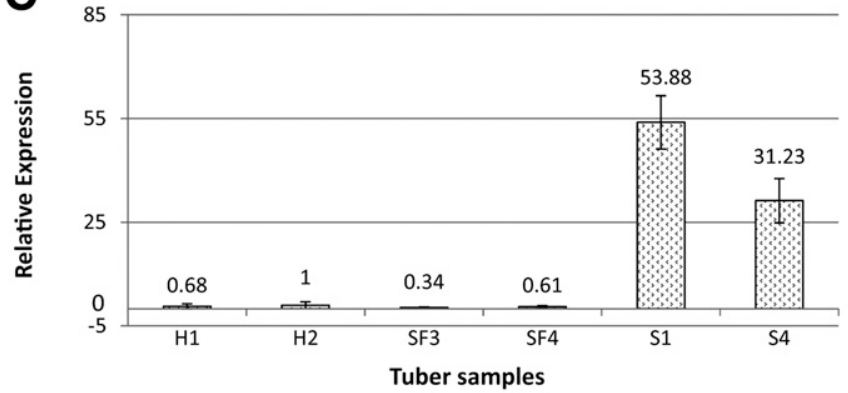

E

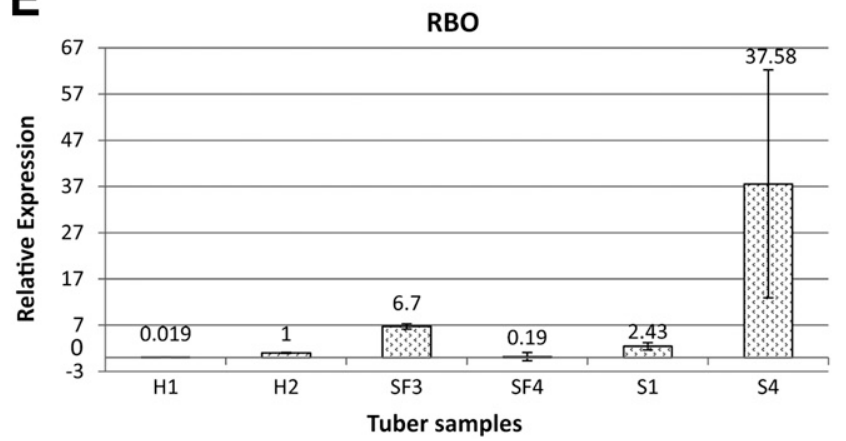

B

Peroxidase

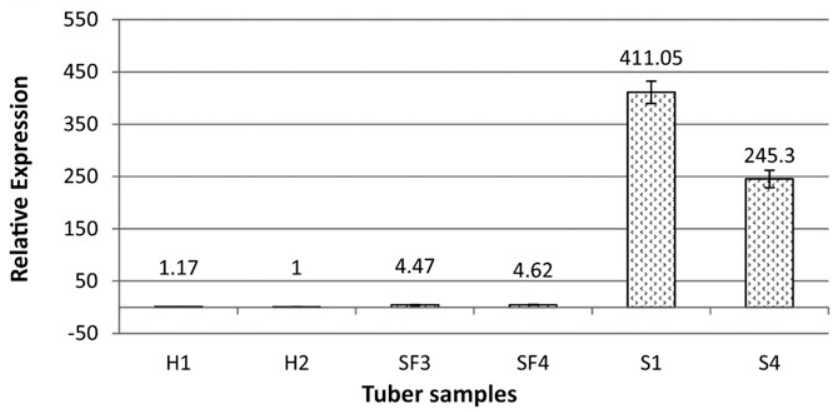

PAR1-c

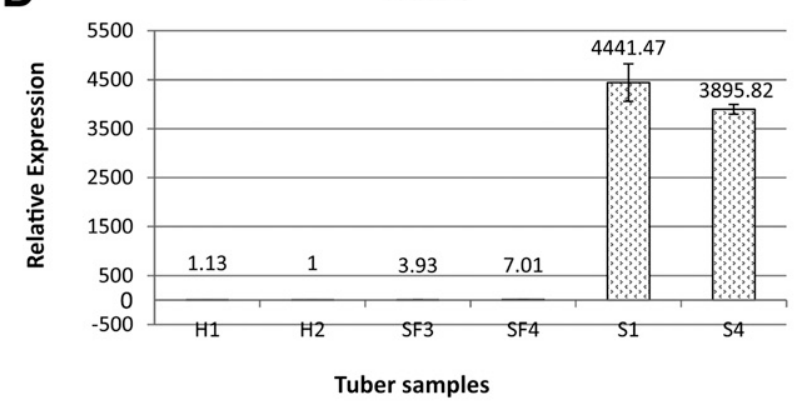

$\mathbf{F}$

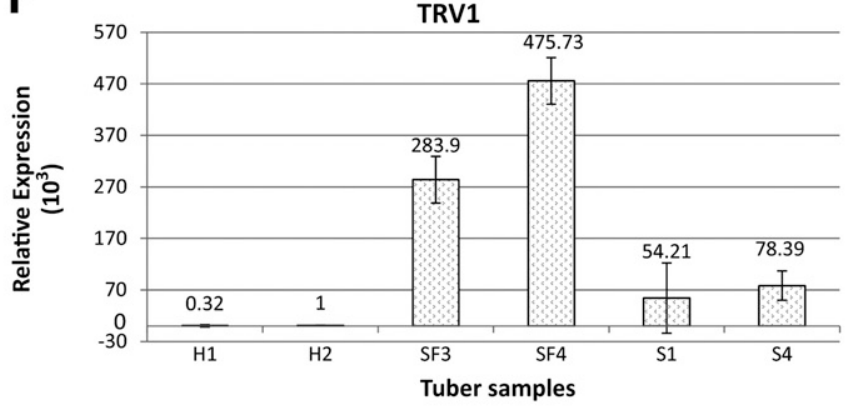

Fig. 2. Validation of microarray data using quantitative reverse transcription-polymerase chain reaction (qRT-PCR). A to E, Relative quantitation of hypersensitive response and biotic stress-related genes selected from the microarray dataset as being differentially expressed. Samples are healthy tuber RNA (H1, H2), infected spraing-free tuber (SF3, SF4), and infected spraing symptomatic (S1, S4). F, Relative quantitation of Tobacco rattle virus (TRV) RNA1. Numbers above each sample bar are relative expression means of three technical repeats. Relative expression was normalized against potato cyclophilin. Bars indicate standard error of $\Delta \Delta \mathrm{CT}$ (cycle threshold) measurements. 
brown-black precipitate; ii) trypan blue, which is excluded from live tissue but can accumulate in dead and dying tissue, such as that affected by PCD; and iii) phloroglucinol, which reacts with coniferyl and sinapyl aldehyde chains of lignin, that associate with suberin during the formation of corky tissue in plants. With DAB staining, the spraing tissue was darkly stained, indicating intense $\mathrm{H}_{2} \mathrm{O}_{2}$ accumulation (Supplementary Fig. S2). The healthy tuber had a lighter, more even staining, which is likely due to activation of peroxidases during mechanical damage as a result of the sectioning of the tuber. Likewise, the brown area of spraing within the affected tuber was strongly stained with trypan blue, indicating accumulation of dead tissue, whereas a spraingfree tuber showed almost no staining. The phloroglucinol staining produced intense pink coloration that was limited to the spraing area, indicative of the corky nature of the spraing.

\section{Spraing formation and reduced TRV load in tuber tissue.}

We also used qRT-PCR to quantitate the level of TRV in the same healthy, spraing-free, and spraing-affected samples. The two spraing-affected samples $(\mathrm{S} 1, \mathrm{~S} 4)$ had a relative mean TRV concentration of 66.3 (S1, 54.2 and S4, 78.3), whereas the spraing-free samples (SF3 and SF4) had a relative mean TRV concentration of 379.8 (SF3, 283.9; SF4, 475.7) (Fig. 2F). Thus, the two spraing-free samples contained at least four times the amount of TRV than did the spraing-symptomatic samples. This suggests that spraing formation may be associated with reduction of virus levels in infected tuber tissue. The HRrelated genes that we examined above were expressed in much higher amounts in spraing-symptomatic tissue (Fig. 2), suggesting that their expression coincides with the initial high accumulation of TRV and that this interaction leads to active tuber defense that results in spraing formation and, subsequently, virus reduction in the tuber.

\section{TRV infection type in spraing-affected tubers.}

TRV infections in potato may be of the NM-type, with RNA1 only, or of the M-type, with RNA1 and RNA2 together. M-type infections produce virus particles that can be nematode-transmitted and are stable during extraction from plants. NM-type infections do not produce virus particles, cannot be nematode-transmitted and are susceptible to degradation during extraction. We collected samples of spraing tissue from ten field-grown 'Pentland Dell' tubers, homogenized them in phosphate buffer and immediately mechanically inoculated the homogenate to Chenopodium quinoa seedlings. This plant species is highly susceptible to viruses and six of the ten tuber homogenates produced infection symptoms on these plants. TRV RNA1 was detected in these six plants by RTPCR but not in the four plants showing no symptoms. Further transfer of TRV from homogenates of the infected Ch. quinoa was successful for two of the six plants, and for these two plants the homogenate remained infectious even after three cycles of freezing at $-20^{\circ} \mathrm{C}$ and thawing at room temperature. This shows that the two surviving isolates are M-type, whereas the four nontransmissible isolates are most likely NM type. One of the M-type isolates reacted in a Western blot with TRV (isolate PRN)-specific antisera (data not shown), whereas the second isolate did not react with PRN-, N5-, SYM-, or Pea early-browning virus SP5-specific antisera and so could not be characterized. This work shows that tubers with spraing symptoms can carry both NM- and M-type TRV infections.

\section{TRV distribution in a spraing-affected tuber.}

To study the association between the relative level of virus and appearance of spraing symptoms in tuber tissue, we examined the distribution of TRV across a single potato tuber showing discrete areas of spraing separated by spraing-free areas (Fig. 3A). Eleven separate areas were excised and RNA was extracted for RT-PCR and qRT-PCR analysis. Eight areas were free of visible spraing symptoms (SF1 to SF8), two areas (S10, S11) had strong spraing symptoms, and one (S9) had a small area of faint spraing. Using RNA1-specific RT-PCR, TRV was strongly amplified from five of the eight spraing-free areas and weakly amplified from a sixth (SF1). All three spraing-affected areas amplified a TRV signal that was noticeably weaker than the majority of the spraing-free areas, suggesting that less virus was present in these areas (Fig. 3B). Five of the samples (SF1, SF6,

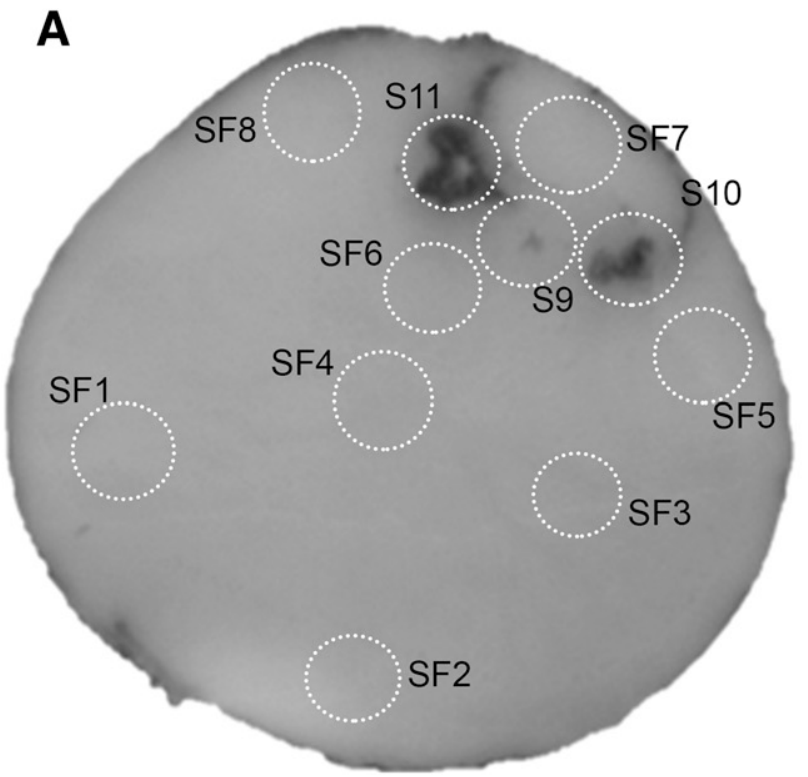

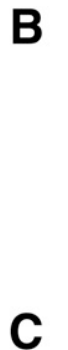

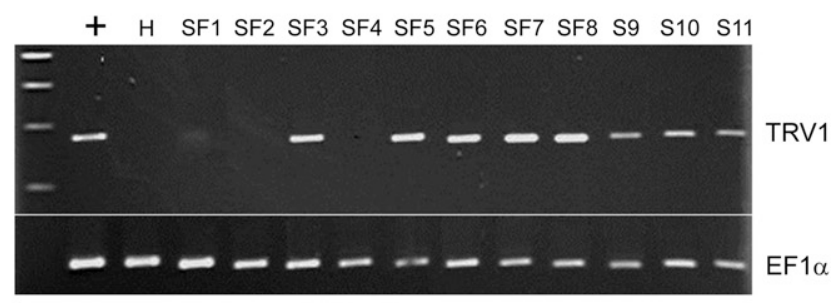

C

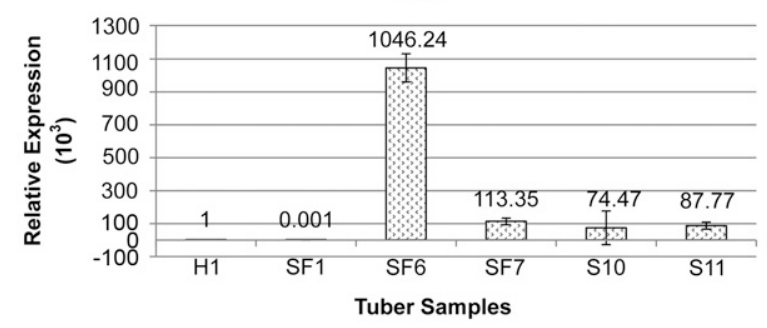

Fig. 3. Distribution of Tobacco rattle virus (TRV) across a potato tuber section. A, Potato tuber section with areas of visible spraing production in the upper right part of the tuber. Named sampled areas are indicated by white dotted circles. B, Reverse transcription-polymerase chain reaction (RT-PCR) of RNA extracted from the different sampled areas. SF denotes visibly spraing-free; S, visible spraing formation. Samples were tested by amplification for TRV RNA1 and for potato EF1 $\alpha$. + denotes a known TRV-infected potato sample; H, a known uninfected potato sample. A DNA ladder appears at the left of the figure. C, Quantitative RT-PCR of TRV RNA1 in selected spraing-symptomatic and spraing-free areas. EF1 $\alpha$ was used as the expression standard. 
SF7, S10, and S11), selected as having different spraing or spraing-free appearances, were tested by qRT-PCR in comparison with a sample from a healthy tuber. This analysis showed that two of the three spraing-free samples had the highest amounts of TRV, with one of them (SF6) containing $>9$ times the amount of virus than the second-highest sample (SF7) and $>12$ times more virus that either of the two spraing-symptomatic samples (S10 and S11) (Fig. 3C.). This is in agreement with the previous results (Fig. 2F), showing that infected spraing-free tuber tissue may have higher levels of virus than does spraing-symptomatic tissue, so that lack of observable spraing in a potato tuber is no guarantee that high levels of virus are not present.

\section{Potato mop-top virus (PMTV) and TRV}

induce similar host responses.

Spraing symptoms are also described in potatoes infected with PMTV, which is a segmented RNA plant virus that is transmitted by the soil organism Spongospora subterranea (Beuch et al. 2015; Nielson and Mølgaard 1997). Although, as with TRV, no detailed investigation of PMTV spraing has been done, it was suggested that TRV is more widespread than PMTV but that PMTV is more predominant in wetter regions (Nicolaisen et al. 1999). We obtained potatoes from a different field trial and identified individual tubers that were either virus-free, infected with TRV, infected with PMTV, or infected with both TRV and PMTV (data not shown). Of 15 virus-infected tubers, eight had visible spraing symptoms and seven were spraing-free. The expression level of two of the genes previously identified as being upregulated during TRV infection (PAR1-c and SP) were examined by qRT-PCR in comparison with their expression in a pooled virus-free tuber sample. As before, PAR1-c and SP gene expression was higher in spraing-symptomatic samples as compared with nonsymptomatic samples (Fig. 4). The increase in PAR1-c and SP expression occurred in both TRV- and PMTVinfected tubers, and infection with both viruses simultaneously did not have an additive effect of the expression of these genes.
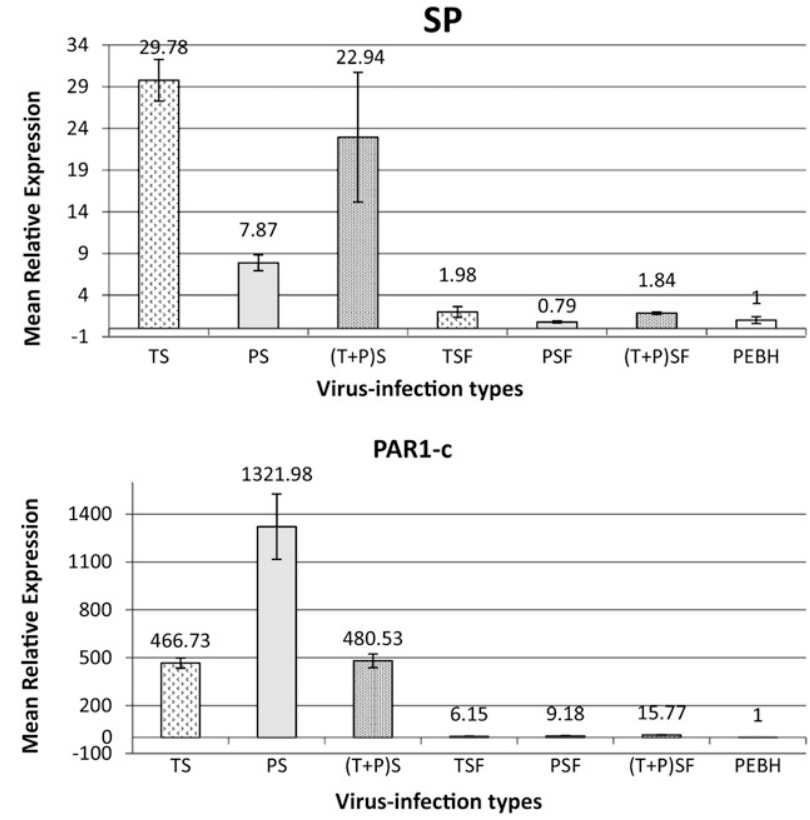

Fig. 4. Potato gene expression induced during spraing induction by Tobacco rattle virus (TRV) and Potato mop-top virus (PMTV). Relative expression levels of two hypersensitive response-related genes (SP and PAR1-c) in response to infection by TRV (T), PMTV (P), and TRV plus PMTV $(\mathrm{T}+\mathrm{P})$. S denotes samples showing spraing symptoms; SF, samples without spraing symptoms. PEBH is a mixed sample from uninfected potato tubers.

\section{DISCUSSION}

Several studies have reported the analysis of host plant gene expression following virus infection; however, none have involved TRV infection of potato (Baebler et al. 2009; FernandezCalvino et al. 2014; Kogovšek et al. 2010; Pompe-Novak et al. 2006; Rodrigo et al. 2012). Furthermore, the studies of virus infection (Potato virus $Y$ ) in potato analyzed only leaves and not tubers.

Here, we report the first molecular experiments undertaken to investigate spraing production in potato tubers in response to virus infection. Gene expression analysis identified a large population of potato tuber genes that are upregulated during TRV infection, including genes known to be induced during pathogen infection, such as those involved in the HR. The increase in expression of a subset of these genes was confirmed by qRT-PCR, and the association of spraing formation with cell death, $\mathrm{H}_{2} \mathrm{O}_{2}$ production, and lignification was further demonstrated by histological staining.

The infection of potato plants by TRV primarily occurs underground when the plant is fed on by viruliferous nematodes, and this process is almost entirely unexamined. Potato tubers are formed from swellings that develop at the end of stolons, which are outgrowths of the stem that are located underground. It would seem possible that infection of the developing stolon tip by TRV might result in a widespread later infection of the tuber in which the virus and associated spraing are located throughout the tuber. If the virus is introduced into the epidermal cells of a more mature tuber, then, it might be expected that the virus and spraing would be located at one side of the tuber, near to the site of introduction. Both of these patterns of spraing are seen in fieldgrown potatoes.

We showed that spraing-symptomatic tubers can be infected with RNA1-only, NM-type infections and with RNA1 plus RNA2, M-type infections. Whether, for NM infections, the separation of RNA1 and RNA2 occurs during the nematode inoculation process or by a selective inhibition of either RNA2 replication, movement, or both in the tuber is not yet known. Interestingly, our results show that high levels of TRV can occur across a wide area of a tuber that does not show spraing symptoms. Whether the virus reaches these areas passively as the tuber expands and matures or whether it actively moves through the tuber vasculature or through plasmodesmata between adjacent cells is, again, not known.

Previously, studies using RT-PCR to detect TRV in potato tubers found that the virus was unevenly distributed (Crosslin and Thomas 1995; Crosslin et al. 1999; Xenophontos et al. 1998). TRV was more reliably detected in areas containing spraing tissue or located just inside a necrotic arc rather than outside of or distant from such an arc. However, no attempt was made to quantify the virus in different parts of the tuber. Using qRT-PCR, we have shown that higher levels of TRV can exist in symptom-free areas of the tuber that are located some distance away from the arc of spraing tissue. The important practical relevance of this finding is that there can be high levels of TRV in tubers of spraing-reactive potato cultivars (e.g., 'Pentland Dell') that do not, at the time of sampling, show visible signs of spraing. This is particularly important if these tubers are selected as seed for subsequent potato cultivation and highlights the need to apply molecular testing rather than simple phenotypic observations for virus-free seed potato production.

We have also shown by qRT-PCR that defense gene expression in 'Pentland Dell' potato, exemplified by PAR1-c and SP in this study, responds similarly to TRV and PMTV. As these viruses are not related in their genome sequences or encoded proteins, these parts of the host defense pathway must be reacting to a generic signal, such as metabolic stress or, perhaps, double-stranded 
RNA accumulation, which is formed as a replication intermediate of many RNA plant viruses. From our TRV studies, we suggest that the formation of spraing is a host reaction to virus infection and multiplication that leads to a reduction in virus levels where spraing develops. Further work is required to ascertain whether spraing formation causes a similar reduction in PMTV in potato tubers.

\section{MATERIALS AND METHODS}

\section{Potato tuber sampling and sample preparation.}

The potato tubers used for the microarray study were provided by F. Dale (Caithness Potato Exports Ltd., Perth, Scotland) from commercial fields in which trichodorid nematodes carrying TRV were known to occur in high numbers. The tubers were from the variety 'Pentland Dell', which is known to produce spraing symptoms, and were collected as part of the end-of-season harvest and were transported to The James Hutton Institute (JHI) in Dundee, U.K., for further examination. Uninfected (healthy) 'Pentland Dell' tubers were obtained from field plots that were known, historically, to be free from TRV disease.

Potato tubers (varieties Electra and Burren) for PMTV testing and analysis were provided by C. Lacomme, Science and Advice for Scottish Agriculture, Edinburgh. Other tubers of mixed varieties were provided by L. Sullivan (JHI, Dundee).

To prepare material for microarray analysis, the 'Pentland Dell' tubers were cut into slices of about $0.5-\mathrm{cm}$ thickness and were examined for the presence of spraing symptoms (brown or black lines and flecks). RNA from each tuber was tested by RTPCR (discussed below) for the presence of TRV. More than 50 small blocks of tissue (about $5 \mathrm{~mm}^{3}$ ) were collected separately from each tuber (concentrating on areas exhibiting spraing symptoms, when present) and were immediately frozen with liquid nitrogen. In total, tuber tissue samples were collected from 12 different, individual tubers so that separate samples from four tubers with spraing symptoms and TRV (samples S1 to S4), four tubers without spraing symptoms but with TRV (spraing-free samples SF1 to SF4), and four tubers without spraing or TRV (healthy samples H1 to H4) were collected for microarray analysis. The tuber samples were immediately freeze-dried, were ground to a powder, and were stored at $-80^{\circ} \mathrm{C}$.

RNA was extracted from the tuber samples using the hot phenol extraction method of Ducreux et al. (2008). After successive precipitations with lithium chloride and then ethanol, the RNA was further cleaned using a Qiagen RNeasy column and treatment with Ambion TURBO DNAse, following the suppliers' instructions. The RNA was quantitated using a Nanodrop 1000 spectrophotometer, and the quality was assessed using an Agilent 2100 Bioanalyzer.

\section{Microarray analysis.}

Experimental design and data can be accessed at ArrayExpress (E-MTAB-4670). Experiments were performed using a customdesigned Agilent gene expression microarray, referred to as the potato oligo chip initiative (POCI) array, which consists of 60mer oligonucleotide probes representing 42,034 potato unigenes in $4 \times 44 \mathrm{~K}$ format (ArrayExpress, A-MEXP-1117). Briefly, potato tuber RNA samples were converted to Cy3-labeled cRNA using the Low-Input quick amp labeling kit (Agilent), following the One-Color microarray-based gene expression analysis v. 6.5 protocol. Labeled cRNAs were hybridized to the POCI arrays overnight and were scanned as recommended, using a G2505B scanner (Agilent). Four replicates of each of the 12 RNA samples (themselves representing four biological replicates of the three tuber types) were processed on the array. Data were extracted from each of the arrays, using Feature Extraction v. 10.7.3.1 software (Agilent) and default parameters, prior to importing into
Genespring v. 7.3 software (Agilent) for normalization, quality control, and analysis. Default normalization (Agilent One-Color) settings were applied and probes that were flagged as 'absent' in $\geq 10$ of 12 samples were removed from the analysis, leaving 27,895 probes. The resulting data were subject to pairwise comparison (volcano plots), applying filters for a Student's $t$ test $\leq 0.01$, and normalized fold-change $\geq 2 \times$, to reveal differential expression of individual genes between contrasting sample sets.

\section{cDNA synthesis for qRT-PCR.}

cDNA was synthesized, using $1 \mu \mathrm{g}$ of purified total RNA and $1 \mu \mathrm{l}$, each, of $100-\mu \mathrm{m}$ oligo $\mathrm{dT}_{17}$ and random hexamer primers with Superscript III reverse transcription (Invitrogen) in a $20-\mu 1$ reaction, as specified by the manufacturer. The synthesis reaction was done for $1 \mathrm{~h}$ at $55^{\circ} \mathrm{C}$, and the cDNA was further treated with RNAse $\mathrm{H}$ to remove RNA, as recommended.

\section{qRT-PCR validation of potato gene expression.}

Validation of the expression profiles of a subset of potato genes identified from the microarray experiments as being differentially expressed between the three treatments (healthy, virus-infected spraing-free, or virus-infected spraing symptomatic) was done by qRT-PCR, using the Power SYBR green master mix (Applied Biosystems). Two RNA samples of each treatment analyzed in the microarray experiments were tested separately by qRT-PCR. A $25-\mu 1$ volume qRT-PCR reaction contained $5 \mu \mathrm{l}$ of $1 / 25$ diluted cDNA, $12.5 \mu \mathrm{l}$ of Power SYBR green master mix, and gene-specific primers. Each primer pair was adjusted to the optimum ratio and concentration, using a pooled sample of all cDNAs, and was analyzed using the software supplied with the StepOnePlus real-time PCR system (Applied Biosystems). Melt curve analysis indicated that the primers all produced a single amplicon, with no evidence of significant amplification of nonspecific products (data not shown). qRT-PCR was repeated three times (technical replicates) for each RNA sample. The two reference genes selected for these experiments, elongation factor 1 alpha $(\mathrm{EF} 1 \alpha)$ and cyclophilin (Cyc), were previously shown to be appropriate controls for gene-expression studies in potato (Hunter et al. 2016; Ross et al. 2011). For the qRT-PCR analyses to validate the microarray results, RNA of sample $\mathrm{H} 2$ was used as the calibrator to calculate relative expression levels, whereas, in the PMTV and TRV experiment, a mixed sample from uninfected potato tubers was used as the calibrator.

\section{Virus detection and quantification.}

Detection of TRV in the tuber RNA samples was done using Illustra Ready To Go (GE Healthcare) beads that carry out a combined RT-PCR reaction. RNA $(5 \mu \mathrm{g})$ was used in a 50- $\mu$ reaction containing primers 1760 (CAGTCTATACACA GAAACAGA) and 1761 (GACGTGTGTACTCAAGGGTT), at a concentration of $400 \mathrm{nM}$. These primers are specific to TRV RNA1, flank the sequence for the $16 \mathrm{~K}$ gene, and amplify a fragment of 463 bp. PMTV was detected in a similar way, using primers PTGB2FOR (CGTCGACAAATGGTCCGGAATAACG AAATTG) and PTGB2REV (CCTCGAGTTAACCTCCATAT GACCTGCAGC), which amplify a 356-bp fragment of the viral TGB2 gene. PCR products were detected by agarose gel electrophoresis and ethidium bromide staining.

qRT-PCR of TRV used RNA1-specific primers 2353 (TA CCAAGGGAATGTGTTCTA) and 2354 (CTCGGAACTCC AGCTATC), which amplify a 91-bp fragment of the viral replicase gene. Analysis was done as described above.

\section{Staining of tuber sections.}

Samples of cut-tuber sections were collected using a $12-\mathrm{mm}$ diameter cork borer and were trimmed to a 2-mm thickness 
with a vibroslicer. Three samples from each tuber were examined in parallel, immediately after sectioning, comparing spraing-bearing and spraing-free tubers collected from the same viruliferous field. Trimmed sections from the two types of tuber were treated with three different histological stains. For trypan blue staining, the sections were washed in distilled water and, then, were boiled with lactophenol trypan-blue solution (10 $\mathrm{mg}$ of trypan-blue, $20 \mathrm{ml}$ of absolute ethanol, $10 \mathrm{ml}$ of

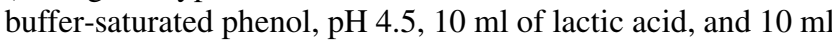
of deionized water) for 2 min (Koch and Slusarenko 1990). The stained tissue sections were then washed with $70 \%$ ethanol, were rinsed in distilled water, and finally, were destained over three weeks in $50 \%$ chloral hydrate solution, with weekly changes of the solution until the background staining was removed. For phloroglucinol staining, the sections were submerged in a $2 \%$ phloroglucinol and $70 \%$ ethanol solution at room temperature for $30 \mathrm{~min}$. To stop the staining reaction, the section was washed in a $2.5 \mathrm{M} \mathrm{HCl}$ solution and then rinsed in distilled water before examination (Liljegren 2010). For DAB staining the sections were immersed using SIGMAFAS DAB tablets (Sigma-Aldrich). To stop the staining reaction, the sections were washed in phosphate buffer before examination (Daudi and O'Brien 2012).

\section{ACKNOWLEDGMENTS}

We would like to thank L. Ducreux for help and advice with potato tuber sample processing. This work was funded by the Scottish Government Rural and Environment Science and Analytical Services (RESAS). G. Sahi received funding from the Merit Scholarship Programme (MSP) of the Islamic Development Bank (IDB).

\section{LITERATURE CITED}

Baebler, S., Krečič-Stres, H., Rotter, A., Kogovšek, P., Cankar, K., Kok, E. J., Gruden, K., Kovač, M., Žel, J., Pompe-Novak, M., and Ravnikar, M. 2009. PVY ${ }^{\text {NTN }}$ elicits a diverse gene expression response in different potato genotypes in the first $12 \mathrm{~h}$ after inoculation. Mol. Plant Pathol. 10:263-275.

Beuch, U., Berlin, S., Akerblom, J., Nicolaisen, M., Nielsen, S. L., Crosslin, J. M., Hamm, P. B., Santala, J., Valkonen, J. P. T., and Kvarnheden, A. 2015. Diversity and evolution of potato mop-top virus. Arch. Virol. 160: 1345-1351.

Crosslin, J. M., and Thomas, P. E. 1995. Detection of tobacco rattle virus in tubers exhibiting symptoms of corky ringspot by polymerase chain reaction. Am. Potato J. 72:605-609.

Crosslin, J. M., Thomas, P. E., and Brown, C. R. 1999. Distribution of tobacco rattle virus in tubers of resistant and susceptible potatoes and systemic movement of virus into daughter plants. Am. J. Potato Res. 76:191-197.

Daudi, A., and O'Brien, J. A. 2012. Detection of hydrogen peroxide by DAB staining in Arabidopsis leaves. Bio Protoc. 2:e263.

Ducreux, L. J. M., Morris, W. L., Prosser, I. M., Morris, J. A., Beale, M. H., Wright, F., Shepherd, T., Bryan, G. J., Hedley, P. E., and Taylor, M. A. 2008. Expression profiling of potato germplasm differentiated in quality traits leads to the identification of candidate flavour and texture genes. J. Exp. Bot. 59:4219-4231.

Fernandez-Calvino, L., Osorio, S., Henandez, M. L., Hamada, I. B., del Toro, F. J., Donaire, L., Yu, A., Bustros, R., Fernie, A. R., MartinezRivas, J. M., and Llave, C. 2014. Virus-induced alterations in primary metabolism modulate susceptibility to Tobacco rattle virus in Arabidopsis. Plant Physiol. 166:1821-1838.
Ghazala, W., and Varrelmann, M. 2007. Tobacco rattle virus $29 \mathrm{~K}$ movement protein is the elicitor of extreme and hypersensitive-like resistance in two cultivars of Solanum tuberosum. Mol. Plant Microbe Interact. 20:1396-1405.

Harrison, B. D., and Robinson, D. J. R. 1978. The tobraviruses. Adv. Virus Res. 23:25-77.

Harrison, B. D., and Robinson, D. J. 1982. Genome reconstitution and nucleic acid hybridisation as methods of identifying particle-deficient isolates of tobacco rattle virus in potato plants with stem mottle disease. J. Virol. Methods 5:255-265.

Hunter, L. J. R., Brockington, S. F., Murphy, A. M., Pate, A. E., MacFarlane, S. A., Palukaitis, P., and Carr, J. P. 2016. RNA-dependent RNA polymerase 1 in potato (Solanum tuberosum) and its relationship to other plant RNA-dependent RNA polymerases. Sci. Rep. 6:23082.

Koch, E., and Slusarenko, A. 1990. Arabidopsis is susceptible to infection by a downy mildew fungus. Plant Cell 2:437-445.

Kogovšek, P., Pompe-Novak, M., Baebler, S., Rotter, A., Gow, L., Gruden, K., Foster, G. D., Boonham, N., and Ravnikar, M. 2010. Aggressive and mild Potato virus $Y$ isolates trigger different specific responses in susceptible potato plants. Plant Pathol. 59:1121-1132.

Liljegren, S. 2010. Phloroglucinol stain for lignin. Cold Spring Harb. Protoc. doi:10.1101/pdb.prot4954

MacFarlane, S. A. 1999. Molecular biology of the tobraviruses. J. Gen. Virol. 80:2799-2807.

Mojtahedi, H., Crosslin, J. M., Santo, G. S., Brown, C. R., and Thomas, P. E. 2001. Pathogenicity of Washington and Oregon isolates of tobacco rattle virus on potato. Am. J. Potato Res. 78:183-190.

Nicolaisen, M., Bösze, Z., and Nielsen, S. L. 1999. Detection of tobacco rattle virus in potato tubers using a simple RT-PCR procedure. Potato Res. 42:173-179.

Nielsen, S. L., and Mølgaard, J. P. 1997. Incidence, appearance and development of potato mop-top furovirus-induced spraing in potato cultivars and the influence on yield, distribution in Denmark and detection of the virus in tubers by ELISA. Potato Res. 40:101-110.

Ploeg, A. T., Brown, D. J. F., and Robinson, D. J. 1992a. Acquisition and subsequent transmission of tobacco rattle virus isolates by Paratrichodorus and Trichodorus nematode species. Neth. J. Plant Pathol 98:291-300.

Ploeg, A. T., Brown, D. J. F., and Robinson, D. J. 1992b. The association between species of Paratrichodorus and Trichodorus vector nematodes and serotypes of tobacco rattle tobravirus. Ann. Appl. Biol. 121:619-630.

Pompe-Novak, M., Gruden, K., Baebler, S., Krečič-Stres, H., Kovač, M., Jongsma, M., and Ravnikar, M. 2006. Potato virus Y induced changes in the gene expression of potato (Solanum tuberosum L.). Physiol. Mol. Plant Pathol. 67:237-247.

Rodrigo, G., Carrera, J., Ruiz-Ferrer, V., Toro, F. J., Llave, C., Voinnet, O., and Elena, S. F. 2012. A meta-analysis reveals the commonalities and differences in Arabidopsis thaliana in response to different viral pathogens. PLoS One 7:e40526.

Ross, H. A., Wright, K. M., McDougall, G. J., Roberts, A. G., Chapman, S. N., Morris, W. L., Hancock, R. D., Stewart, D., Tucker, G. A., James, E. K., and Taylor, M. A. 2011. Potato tuber pectin structure is influenced by pectin methyl esterase activity and impacts on cooked potato texture. J. Exp. Bot. 62:371-381.

Vassilakos, N., Vellios, E. K., Brown, E. C., Brown, D. J. F., and MacFarlane, S. A. 2001. Tobravirus 2b protein acts in trans to facilitate transmission by nematodes. Virology 279:478-487.

Xenophontos, S., Robinson, D. J., Dale, M. F. B., and Brown, D. J. F. 1998 Evidence for persistent, symptomless infection of some potato cultivars with tobacco rattle virus. Potato Res. 41:255-265.

\section{AUTHOR-RECOMMENDED INTERNET RESOURCE}

ArrayExpress database:http://www.ebi.ac.uk/arrayexpress 